The Astrophysical Journal, 228: L93-L98, 1979 March 1

(c) 1979. The American Astronomical Society. All rights reserved. Printed in U.S.A.

\title{
CALCIUM ISOTOPIC ANOMALIES AND THE LACK OF ALUMINUM-26 IN AN UNUSUAL ALLENDE INCLUSION
}

\author{
Typhoon Lee, ${ }^{*}$ William A. Russell, $\dagger$ and G. J. Wasserburg $\dagger$ \\ Received 1978 October 31; accepted 1978 November 28
}

\begin{abstract}
We have studied the $\mathrm{Mg}$ and $\mathrm{Ca}$ isotopic compositions of an unusual Allende inclusion dominated by hibonite, which is the most refractory and possibly the most primitive major oxide mineral. No ${ }^{26} \mathrm{Mg}$ excess was found in spite of the high ${ }^{27} \mathrm{Al} /{ }^{24} \mathrm{Mg}\left(1>10^{3}\right)$ of some samples, indicating an initial $\left({ }^{26} \mathrm{Al} /{ }^{27} \mathrm{Al}\right)_{0}<2 \times 10^{-7}$, a factor of 250 less than found in some other Allende inclusions. The upper limit for $\mathrm{Mg}$ isotopic fractionation is $20 \%$ per amu. Anomalous but uniform $\mathrm{Ca}$ isotopic compositions were found for bulk samples of coexisting phases and microscopic grains. The $\mathrm{Ca}$ anomaly is a superposition of a large mass-dependent fractionation effect of $7.5 \%$ per amu favoring the heavy isotopes and small $(1 \%-2 \%$ ) "nonlinear" effects of presumably nuclear origin. If the lack of ${ }^{26} \mathrm{Al}$ is due to a time delay of $6 \times 10^{6} \mathrm{yr}$ for the formation of the hibonite inclusion, then condensation models require modification. The $\mathrm{Ca}$ effects suggest the alternative that ${ }^{26} \mathrm{Al}$ was not uniformly distributed in the solar system. These results accentuate the curious and unexplained association between large mass fractionation and nuclear effects. They also reinforce the scenario which envisages an early solar system consisting of isotopically and chemically distinct reservoirs resulting from the incomplete mixing of several nucleosynthetic components. It is not evident whether these components originated within the solar system or from another star.
\end{abstract}

Subject headings: abundances - meteors and meteorites - nucleosynthesis - solar system: general

\section{INTRODUCTION}

Extensive searches for evidence of heterogeneity in the early solar system due to incomplete mixing of material produced in different nucleosynthetic environments have yielded positive results over the past few years. Two small inclusions in the Allende meteorite were recently found which had large isotopic anomalies in many elements consisting of both fractionation and of enrichments and depletions in individual isotopes. It was recognized that a more complete picture could emerge only as further examples of such anomalous material were discovered. In this Letter we report the discovery of a third such inclusion from the Allende meteorite which shows large $\mathrm{Ca}$ isotopic anomalies and the absence of $\mathrm{Mg}$ isotopic effects. This inclusion has a remarkable mineralogical and chemical composition and is rich in hibonite. Among the minerals in $\mathrm{Ca}-\mathrm{Al}-$ rich inclusions, hibonite $\mathrm{Ca}(\mathrm{Al}, \mathrm{Ti}, \mathrm{Mg})_{12} \mathrm{O}_{19}$ has the highest estimated condensation temperature for major element oxides from the solar nebula (Blander and Fuchs 1975). Since this phase may represent a very early condensate and may be chemically resistant, it may have a good chance of exhibiting isotopic anomalies. Furthermore, hibonite is rich in $\mathrm{Al}$ and can be poor in $\mathrm{Mg}$, so that the $\mathrm{Mg}$ isotopic effect due to the decay of ${ }^{26} \mathrm{Al}$ could be enhanced. Therefore hibonite has been early recognized as a candidate in the search for anomalies. However, hibonite is usually a minor

* Enrico Fermi Institute, University of Chicago; Robert R. McCormick Postdoctoral Fellow.

† Lunatic Asylum, Division of Geological and Planetary Sciences, California Institute of Technology; Contribution No. $3164(281)$. phase which appears as tiny $(\leq 30 \mu \mathrm{m})$ crystals and is not easily accessible for isotopic analysis. Furthermore, the $\mathrm{Mg}$ content in Allende hibonite is usually fairly high $(0.5 \%-3 \%)$, which would reduce the enhancement in ${ }^{26} \mathrm{Mg}$ (Keil and Fuchs 1971). The present inclusion contains large $(\sim 400 \mu \mathrm{m})$ crystals of hibonite with low $\mathrm{Mg}$ contents $(\leq 0.05 \%)$. Thus extremely large ${ }^{26} \mathrm{Mg}$ excesses were anticipated. If this sample had an initial $\left({ }^{26} \mathrm{Al} /{ }^{27} \mathrm{Al}\right)_{0} \sim 5 \times 10^{-5}$ (Lee, Papanastassiou, and Wasserburg 1976a,b, 1977a, hereafter LPW and years; Wasserburg, Lee, and Papanastassiou 1976, hereafter WLP; Papanastassiou, Lee, and Wasserburg 1977, hereafter PLW), then half of the ${ }^{26} \mathrm{Mg}$ would be from ${ }^{26} \mathrm{Al}$ decay. We studied the $\mathrm{Mg}$ and $\mathrm{Ca}$ isotopic compositions of this hibonite-rich inclusion to investigate the distribution of ${ }^{26} \mathrm{Al}$ in the solar system and to extend the search for isotopic anomalies to this sample of the putatively earliest condensate.

\section{RESULTS}

This inclusion was discovered by one of us (T.L.) in an extended search for large, low $\mathrm{Mg}$ hibonite samples. Dr. E. Anders generously allowed him to excavate selected inclusions from large fragments of the Allende meteorite which were being prepared in his laboratory. One sample proved to contain a cluster of hibonite crystals and was named HAL (Hibonite ALlende). HAL is a subspherical object consisting of two major textural units: a hard coarse-grained interior, and a layered loosely compacted fine-grained exterior. The interior consists of three large single crystals of hibonite in the form of hexagonal plates 
isurrounded by a black rim. The hibonite is close to , $\mathrm{CaAl}_{12} \mathrm{O}_{19}$ with only trace amounts of $\mathrm{Sc}, \mathrm{Ti}, \mathrm{Mg}$, ' and Fe. Each of the crystals consists of a clear rim

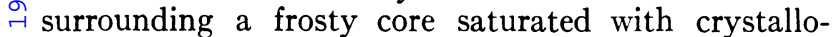
graphically oriented needles of a $\mathrm{Ca}$-Ti-rich phase. The cleavage plates (0001) are dotted with hexagonal pits possibly due to a back reaction process, and small amounts of alkali-rich alteration product had penetrated between the plates. The black rim surrounding the hibonite may be a reaction product. Its chemical composition $\left(57 \% \quad \mathrm{Al}_{2} \mathrm{O}_{3}, 30 \% \mathrm{FeO}, 7 \% \quad \mathrm{SiO}_{2}, 2 \%\right.$ $\left.\mathrm{CaO}, 2 \% \quad \mathrm{Na}_{2} \mathrm{O}, 0.8 \% \quad \mathrm{TiO}_{2}, 0.3 \% \mathrm{MgO}\right)$ does not resemble any known mineral and shows no well defined $\mathrm{X}$-ray diffraction pattern. The fine-grained exterior consists of layers of distinct mineral assemblage which grade out into the typical Allende matrix. The outer parts of the friable fine-grained exterior appear to have been mechanically disrupted during aggregation of the meteorite. The exterior shows some similarities to the much thinner rims in usual inclusions (Wark and Lovering 1977). Petrographic and chemical studies of HAL will be presented elsewhere (Allen et al. 1979; Davis et al. 1979). Clear hibonite grains (1 and 2) were analyzed by the direct loading technique (LPW 1977b). Three batches of hibonite grains $(3,4$, and 5$)$ were crushed $(<50 \mu \mathrm{m})$, leached in a mixture of $\mathrm{HF}$ and $\mathrm{HNO}_{3}$ to remove attached silicates, and then decomposed using $\mathrm{K}_{2} \mathrm{~S}_{2} \mathrm{O}_{7}$ fusion (LPW 1977b). One grain of the black rim was directly loaded and analyzed. Several grains of the black rim were dissolved using $\mathrm{HF}$ $\mathrm{HNO}_{3}$. A sample of the fine exterior in an area immediately adjacent to the black rim and including several layers was dissolved in $\mathrm{HF}$ and $\mathrm{HNO}_{3}$ and analyzed.

Magnesium analytical procedures were previously described (Lee and Papanastassiou 1974, hereafter LP; LPW 1977b). For Ca we followed the procedure described by Russell, Papanastassiou, and Tombrello (1978, hereafter RPT). Each dissolved sample was split into two aliquots. The smaller one $(\sim 10 \%)$ was mixed with a ${ }^{25} \mathrm{Mg}$ spike and a ${ }^{42,48} \mathrm{Ca}$ double spike and passed through an ion exchange column to separate $\mathrm{Mg}$ and $\mathrm{Ca}$ portions. The remaining large aliquot for isotopic composition determination was passed through a different column. $\mathrm{Mg}$ and $\mathrm{Ca}$ blanks of the entire procedure including fusion and one column pass are $22 \mathrm{ng}$ and $140 \mathrm{ng}$, respectively. Most of the blank can be attributed to $\mathrm{K}_{2} \mathrm{~S}_{2} \mathrm{O}_{7}$. The column blank is $1-2 \mathrm{ng}$ for $\mathrm{Mg}$ and $15 \mathrm{ng}$ for $\mathrm{Ca}$. The $\mathrm{Mg}$ added during fusion is comparable to the $\mathrm{Mg}$ in the hibonite sample. Two $\mathrm{Mg}$ blanks agreed within 5\%. For hibonite, the $\mathrm{Al} / \mathrm{Mg}$ ratio was determined by combining the $\mathrm{Ca} / \mathrm{Mg}$ ratio measured for the concentration aliquot and the known stoichiometric ratio $\mathrm{Al} / \mathrm{Ca}=$ 12. The $\mathrm{Al} / \mathrm{Mg}$ reported for the hibonite sample includes the $\mathrm{Mg}$ blank introduced during sample processing. For the black rim the same method was used, except that the average of the slightly variable $\mathrm{Al} / \mathrm{Ca}$ ratio based on several electron probe microanalyses was used. An upper limit of 50 for $\mathrm{Al} / \mathrm{Mg}$ in the exterior can be established by assuming that it contains only $\mathrm{Mg}$ and $\mathrm{Al}$.

The $\mathrm{Ca}$ and $\mathrm{Mg}$ isotopic compositions of various phases of HAL have been analyzed by using the Lunatic I mass spectrometer (Wasserburg et al. 1969). The $\mathrm{Mg}$ data are reported in Table 1 as $\delta^{26} \mathrm{Mg}$, the fractional deviation from the normal value of the ratio ${ }^{26} \mathrm{Mg} /{ }^{24} \mathrm{Mg}$. The data have been corrected for mass fractionation effects by normalizing ${ }^{25} \mathrm{Mg} /{ }^{24} \mathrm{Mg}$ to a standard (Schramm, Tera, and Wasserburg 1970) to determine the fractionation $(f)$ and then using a power law $(1+f)^{2}$ to correct the measured ${ }^{26} \mathrm{Mg} /{ }^{24} \mathrm{Mg}$ ratios. All samples studied show $\delta^{26} \mathrm{Mg}$ consistent with the normal value within uncertainties. Quantitative

TABLE 1

Magnesium Isotopic Results

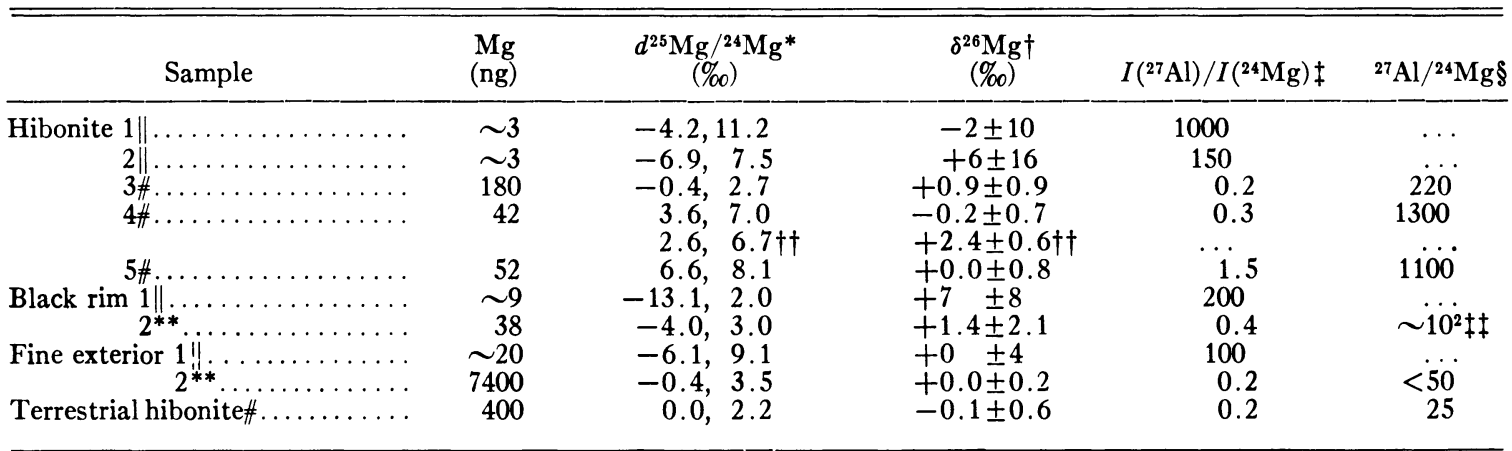

* Permil range of raw measured ${ }^{25} \mathrm{Mg} /{ }^{24} \mathrm{Mg}$ relative to 0.12475 .

+ Permil deviation of ${ }^{26} \mathrm{Mg} /{ }^{24} \mathrm{Mg}$ relative to 0.139805 after correcting for fractionation by normalizing ${ }^{25} \mathrm{Mg} /{ }^{24} \mathrm{Mg}$ to 0.12663 ; errors are 2 standard deviations.

$\ddagger$ Ratio of intensities during analysis.

$\$ \mathrm{Al}$ concentration is from $\mathrm{Al} / \mathrm{Ca} \equiv 12 ; \mathrm{Ca}$ and $\mathrm{Mg}$ concentration are determined by isotopic dilution.

Directly loaded crystals.

\# Fused and chemically separated samples.

** HF dissolved and chemically separated samples.

t† Same sample load reanalyzed in Lunatic III.

$\ddagger \ddagger$ Assuming $\mathrm{Al} / \mathrm{Ca}=24$, the average of EPMA analyses. 
limits for $\left({ }^{26} \mathrm{Al} /{ }^{27} \mathrm{Al}\right)_{0}$ can best be established for hibonites 4 and 5 which have the highest ${ }^{27} \mathrm{Al} /{ }^{24} \mathrm{Mg}$. Both samples have measured $\delta^{26} \mathrm{Mg} \sim 0$, but the ${ }^{27} \mathrm{Al}$ signal was somewhat high for hibonite 5 analysis indicating a possible bias of $-1.5 \%$ in the measured $\delta^{26} \mathrm{Mg}$ (LPW 1977b) and, for hibonite 4 , the reanalysis of the same sample load in the Lunatic III mass spectrometer gave a discrepant $\delta^{26} \mathrm{Mg} \sim 2 \%$. Therefore we adopt a limit on ${ }^{26} \mathrm{Mg}$ excess of $2 \%$, which, combined with the ${ }^{27} \mathrm{Al} /{ }^{24} \mathrm{Mg} \sim 1200$, corresponds to an upper limit on the initial $\left({ }^{26} \mathrm{Al} /{ }^{27} \mathrm{Al}\right)_{0}$ for $\mathrm{HAL}$ of $2 \times 10^{-7}$. In Table 1 we also show the range of deviations of the measured ${ }^{25} \mathrm{Mg} /{ }^{24} \mathrm{Mg}$ ratios $\left(d^{25} \mathrm{Mg}\right)$ during each analysis relative to the mean ratio of normal standards. These deviations reflect mass fractionation effects in the laboratory and in nature. The laboratory fractionation effects on $\mathrm{Mg}$ have a well-defined range of $\sim \pm 3 \%$ for samples of standard size $(\sim 300 \mathrm{ng}$; LPW 1976a). Values well outside this range have been attributed to fractionation effects intrinsic to the samples. Taking into account the smaller size of samples ( $\sim 30 \mathrm{ng})$ used for this study, we can set an upper limit of $\sim 10 \%$ o per mass unit for fractionation effects in the sample-blank mixture. This is the limit on the fractionation in all samples except the hibonite. Since the $\mathrm{Mg}$ blank is $\sim 50 \%$ of the total $\mathrm{Mg}$ in the hibonite samples, the limit for the fractionation intrinsic to the hibonite is $20 \%$ per amu.

The $\mathrm{Ca}$ results were obtained by the double-spike method, using spiked aliquots of each sample to determine the instrumental fractionation and the unspiked remainder to determine the isotopic composition (RPT). Calcium data are reported as deviations $\Delta_{i}$ defined by $\Delta_{i} \equiv\left[\left(R_{i, 44} / R_{i, 44}{ }^{0}\right)-1\right] \times 10^{3}$ where $R_{i, 44}$ is the ratio of isotope $i$ to ${ }^{44} \mathrm{Ca}$ when corrected for laboratory fractionation and $R_{i, 44^{0}}$ is the ratio for normal Ca (RPT). The correction for laboratory fractionation on $R_{i, 44}$ was calculated using the exponential fractionation law from the ${ }^{40} \mathrm{Ca} /{ }^{44} \mathrm{Ca}$ data measured during the composition run and the reference value of ${ }^{40} \mathrm{Ca} /{ }^{44} \mathrm{Ca}$ in the sample determined by the double-spike run. The resulting $\Delta_{i}$ represent the fractional differences of the isotopic ratios corrected only for instrumental effects and indicate shifts in isotopic abundance due to natural fractionation and/or nuclear effects. The $\mathrm{Ca}$ isotopic composition was determined for "bulk" and microscopic samples of hibonite, black rim, and fine-grained exterior. The results (Table 2) show that all samples have large shifts for all isotopes and indicate that these samples are highly anomalous. The $\Delta_{i}$ values for different samples are in reasonable agreement but are not identical within errors, with $\Delta_{40}$ ranging from -30 to -26 . There appears to be a correlation between $\Delta_{40}$ and the amount of Ca processed. As controls, terrestrial $\mathrm{Ca}$ and a total rock sample of Allende were analyzed and found to be identical. We also tabulate the previous results on C1, EK1-4-1, and WA in the same form for the purpose of a consistent and comprehensive discussion. From Figure 1 it is evident that HAL shows a regular shift in $\Delta_{i}$ as a function of mass which is approximately linear. The slope is $7.5 \%$ per amu in favor of the heavy masses. From these data we conclude that the $\mathrm{Ca}$ in $\mathrm{HAL}$ has undergone extreme isotopic fractionation.

It is important to establish whether the deviations $\Delta_{i}$ are solely the result of mass fractionation or if they also show evidence of "nonlinear" effects. Let us assume that the observed value of $\Delta_{40}$ is the result of mass fractionation of normal material (with original isotopic ratios $R_{i, 44^{0}}$ ) following law $A$. Then with the assumed law we may calculate the values ${ }^{A} \Delta_{i, 44}$ for all isotopes which the fractionated normal $\mathrm{Ca}$ would have if it had been shifted so that ${ }^{A} \Delta_{40}{ }^{0}=\Delta_{40}$ (note $\Delta_{44} \equiv 0$ here). A graph of ${ }^{A} \Delta_{i, 44}{ }^{0}$ is shown in Figure 1 for both linear $(\mathrm{L})$ and Rayleigh (R) laws (Rayleigh 1896). Deviations from the fractionation curve ${ }^{A} \Delta_{i, 44}{ }^{0}$ are defined as ${ }^{A} \delta_{i}$ (i.e., ${ }^{A} \delta_{i} \equiv \Delta_{i}-{ }^{A} \Delta_{i, 44}{ }^{0}$ ). Inspection of $\delta_{i}$ in Table 2 and Figure 1 shows that all samples of HAL have a distinct "nonlinear" effect at mass 42 and possibly 43 . Effects may also be present at 48 but are highly dependent on the law used. Note that the deviations at 42 and 43 are preserved independently of our choice of the law. As a further check we normalized the raw composition data to a standard ${ }^{40} \mathrm{Ca} /{ }^{44} \mathrm{Ca}$ value using a linear fractionation law. This resulted in similar effects, demonstrating convincingly that the deviations are not artifacts due to the exponential law normalization to the reference values $R_{i, 44}{ }^{0}$. From the $\delta_{42}$ values we conclude that there exist nonlinear effects in $\mathrm{HAL}$ in addition to the large fractionation effects. The extent to which the $\delta_{i}$ values are precisely given for such highly fractionated material must remain an open question. Furthermore, since there is no a priori way to choose the base isotopes $\left({ }^{40} \mathrm{Ca}\right.$ and ${ }^{44} \mathrm{Ca}$ were used), $\delta_{i}$ obtained through the above decomposition are not unique. A change of base would lead to different nonlinear effects which are related to the present results through a transformation formula similar to that given by McCulloch and Wasserburg (1978).

\section{DISCUSSION}

The $\mathrm{Mg}$ isotopic studies of the unusual Allende inclusion HAL showed that: (1) no $\mathrm{Mg}$ isotopic anomalies were present; (2) the initial $\left({ }^{26} \mathrm{Al} /{ }^{27} \mathrm{Al}\right)_{0}$ for HAL when it crystallized was less than $2 \times 10^{-7}$; and (3) $\mathrm{Mg}$ mass fractionation effect in HAL must be less than $\sim 20 \%$ per amu for the hibonite and 10\%o per amu for other phases. Calcium studies revealed that: (1) large $\mathrm{Ca}$ mass fractionation effects of $\sim 7.5 \%$ per amu were present; (2) additional small "nonlinear" effects of presumably nuclear origin at the level of $\sim 1 \%$ o- $2 \%$ o were present in at least ${ }^{42} \mathrm{Ca}$; and (3) nearly identical $\mathrm{Ca}$ fractionation and nonlinear effects were found in macroscopic and microscopic samples of hibonite and in the adjacent layers containing much lower temperature mineral assemblages.

Although the presence of ${ }^{26} \mathrm{Al}$ in the early solar system seems to be reasonably well established (LPW $1977 a)$, data for $\left({ }^{26} \mathrm{Al} /{ }^{27} \mathrm{Al}\right)_{0}$ are still quite limited. In three Allende inclusions $\left({ }^{26} \mathrm{Al} /{ }^{27} \mathrm{Al}\right)_{0} \sim 5 \times 10^{-5}$ have been found using high-precision mass spectrometry (LPW 1976a,b, 1977a). Ion probe microanalysis (IPMA) confirmed this value for another Allende anorthite crystal (Hutcheon et al. 1978) and less 
TABLE 2

CALCium Isotopic Results

\begin{tabular}{|c|c|c|c|c|c|c|c|c|c|c|c|}
\hline Sample & $\mathrm{Ca}(\mu \mathrm{g})$ & $\Delta_{40}{ }^{*}$ & $\Delta_{42}^{\prime}$ & $\Delta_{42}$ & $\Delta_{43}$ & $\Delta_{46}$ & $\Delta_{48}$ & ${ }^{\mathrm{L}} \delta_{42} \dagger$ & ${ }^{L} \delta_{43}$ & $\mathrm{~L}_{\delta_{46}}$ & $\mathbf{L}_{\boldsymbol{\delta}_{48}}$ \\
\hline $\begin{array}{l}\mathrm{HAL} \\
\quad \text { Exterior } 2 .\end{array}$ & 17.7 & $-30.0 \pm 0.2$ & $-16.4 \pm 0.5$ & $-16.4 \pm 0.2$ & $-8.5 \pm 0.9$ & $+13 \pm 7$ & $+28.1 \pm 0.8$ & $\begin{array}{l}-1.4 \\
(-1.8)\end{array}$ & $\begin{array}{l}-1.0 \\
(-1.3)\end{array}$ & $\begin{array}{l}-2 \\
(-1)\end{array}$ & $\begin{array}{l}-1.9 \\
(+1.2)\end{array}$ \\
\hline $\begin{array}{r}\text { Black rim } 2 \ldots \ldots \\
\text { Hibonite } 4 \ldots \ldots \ldots \\
5 \ldots \ldots \ldots \\
1 \ddagger \ldots \ldots \\
2 \ddagger \ldots \ldots \\
\text { EK1-4-1 split SCA } \$ \\
\end{array}$ & $\begin{aligned} & 0.28 \\
& 6.2 \\
& 6.5 \\
& \sim 0.2 \\
& \sim 0.2\end{aligned}$ & $\begin{array}{c}-26.0 \pm 0.7 \\
-26.6 \pm 0.4 \\
-27.8 \pm 0.3 \\
\text { n.d. } \\
=-30.0 \\
+7.3 \pm 0.1\end{array}$ & $\begin{array}{c}\text { n.d. } \\
-15.4 \pm 0.5 \\
-15.5 \pm 0.5 \\
\text { n.d. } \\
\text { n.d. } \\
+5.2 \pm 0.6\end{array}$ & $\begin{array}{c}\text { n.d. } \\
-15.2 \pm 0.3 \\
-15.8 \pm 0.3 \\
\equiv-16.4 \\
-16.5 \pm 0.3 \\
+5.3 \pm 0.2\end{array}$ & $\begin{aligned} & \text { n.d. } \\
&-7.7 \pm 0.5 \\
&-8.2 \pm 0.5 \\
&-6.8 \pm 1.1 \\
& \text { n.d. } \\
&+2.6 \pm 0.6\end{aligned}$ & $\begin{array}{l}\text { n.d. } \\
\text { n.d. } \\
\text { n.d. } \\
+2 \pm 12 \\
\text { n.d. } \\
-26 \pm 13\end{array}$ & $\begin{array}{c}\text { n.d. } \\
+24.4 \pm 0.7 \\
+24.0 \pm 0.7 \\
\text { n.d. } \\
\text { n.d. } \\
+6.4 \pm 0.6\end{array}$ & $\begin{array}{l}\text { n.d. } \\
-1.9 \\
-1.9 \\
\text { n.d. } \\
-1.5 \\
+1.7 \\
(+1.6)\end{array}$ & $\begin{array}{l}\text { n.d. } \\
-1.0 \\
-1.3 \\
+1.4 \\
\text { n.d. } \\
+0.8 \\
(+0.8)\end{array}$ & $\begin{array}{l}\text { n.d. } \\
\text { n.d. } \\
\text { n.d. } \\
-10 . \\
\text { n.d. } \\
-22\end{array}$ & $\begin{array}{l}\text { n.d. } \\
-2.2 \\
-3.8 \\
\text { n.d. } \\
\text { n.d. } \\
+13.7 \\
(+12.6)\end{array}$ \\
\hline $\begin{array}{l}\text { C1 split } \mathrm{S} 1 \S \ldots \\
\text { WA anorthite } \$ . . . \\
\text { Allende total rock. }\end{array}$ & & $\begin{array}{l}-1.2 \pm 0.2 \\
+3.0 \pm 0.1 \\
+0.2 \pm 0.2\end{array}$ & $\begin{array}{l}-0.8 \pm 0.3 \\
+1.2 \pm 0.4 \\
+0.1 \pm 0.6\end{array}$ & $\begin{array}{l}-0.4 \pm 0.2 \\
+1.3 \pm 0.2 \\
+0.2 \pm 0.3\end{array}$ & $\begin{array}{l}-0.6 \pm 0.5 \\
+0.9 \pm 0.3 \\
+0.2 \pm 0.6\end{array}$ & $\begin{array}{l}-13+7 \\
-13 \pm 13 \\
\text { n.d. }\end{array}$ & $\begin{array}{l}-1.7 \pm 0.2 \\
-2.9 \pm 0.5 \\
-0.2 \pm 0.6\end{array}$ & $\begin{array}{r}+0.2 \\
-0.2 \\
0.1\end{array}$ & $\begin{array}{r}-0.3 \\
0.1 \\
0.2\end{array}$ & $\begin{array}{l}-14 \\
-12 \\
\text { n.d. }\end{array}$ & $\begin{array}{r}-2.9 \\
+0.1 \\
0.0\end{array}$ \\
\hline
\end{tabular}

${ }^{*} \Delta_{i} \equiv\left[\left(R_{i, 44^{c}}{ }^{2} R_{i, 44^{0}}\right)-1\right] \times 10^{3}$ where $R_{i, 44^{c}}$ is the ratio of isotope $i$ to ${ }^{44} \mathrm{Ca}$ corrected for laboratory fractionation effects using the double-spike method; $R_{i, 44^{0}}$ is the corresponding normal

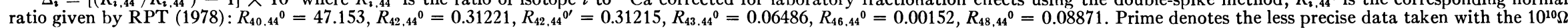
resistor; $\mathrm{n}$ d $=$ not determined; errors are 2 standard deviations; no blank correction has been applied.

$\dagger$ "Nonlinear" effect obtained assuming the linear fractionation law and $\left({ }^{40} \mathrm{Ca},{ }^{44} \mathrm{Ca}\right)$ as base; i.e., ${ }^{\mathrm{L}} \delta_{i}=\Delta_{i}-\left(\Delta_{40}-\Delta_{44}\right)[(\mathrm{i}-44) /(40-44)] ;$ values in parentheses are ${ }^{\mathrm{R}} \delta_{i}$ calculated using the Rayleigh fractionation law; i.e., ${ }^{\mathrm{R}} \delta_{i}=\Delta_{i}-10^{3} \times\left[-1+h^{((44 / i) 1 / 2-1)]}\right]$ where $h \equiv\left(1+\Delta_{40} / 10^{3}\right)^{1 /[(44 / 40) 1 / 2-1]}$; using these definitions the error for $\delta_{i}$ (not shown) can be calculated from errors for $\Delta_{i}$ and $\Delta_{40}$

Directly loaded crystals.

$\S$ Previously published data (LPW 1978) are ${ }^{E} \delta_{i}$ obtained using the exponential fractionation law which gives values very similar to ${ }^{\mathrm{L}} \delta_{i}$ and ${ }^{\mathrm{R}} \delta_{i}$ 


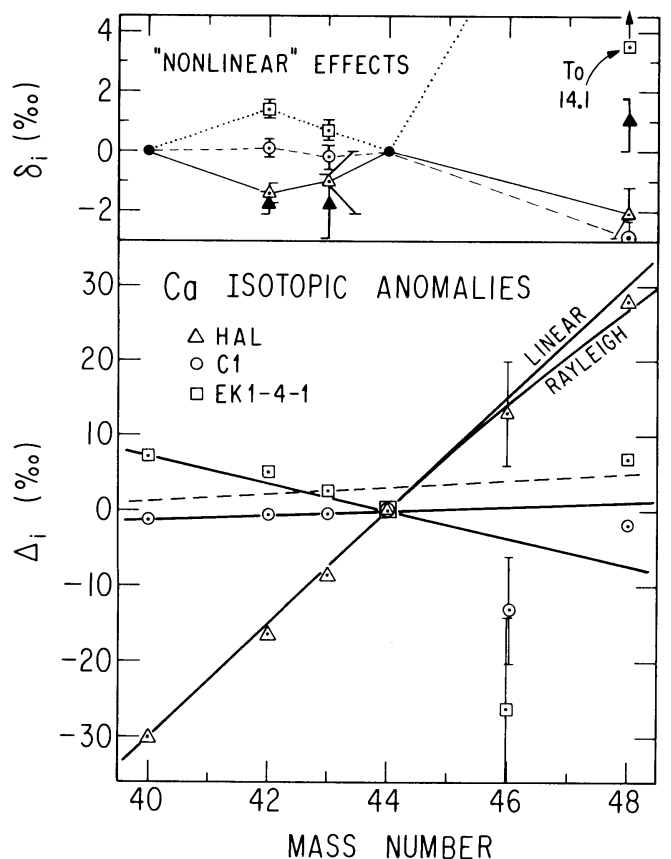

FIG. 1.-Fractional differences of $\mathrm{Ca}$ isotopic ratios relative to normal (corrected for laboratory fractionation). Solid lines, fractionation curves through base isotopes ${ }^{40} \mathrm{Ca}$ and ${ }^{44} \mathrm{Ca}$. "Linear" and "Rayleigh" refer to different fractionation laws. For EK1-4-1 and C1 there is little difference between these two laws. Deviations from the linear curve, $\mathrm{L}_{i}$, due to "nonlinear" effects of presumably nuclear origin are plotted in the upper panel. Solid triangles, ${ }^{\mathrm{R}} \delta_{i}$ for HAL relative to the Rayleigh curve. The broken line in the lower panel is an arbitrary but permissible choice of fractionation curve for EK1-4-1 and illustrates the major changes in the nuclear effects (nonnegative $\delta$ 's for all isotopes except ${ }^{44} \mathrm{Ca}$ ) which can result from a different decomposition procedure.

precise IPMA data on three more Allende inclusions and one inclusion for the Leoville meteorite also yield $\left({ }^{26} \mathrm{Al} /{ }^{27} \mathrm{Al}\right)_{0}$ consistent with this value within broad error limits (Bradley, Huneke, and Wasserburg 1977; Lorin and Christophe Michel-Levy 1978). These ratios of $\sim 5 \times 10^{-5}$ are based primarily on data for anorthite and grossular which have condensation or "appearance" temperatures several hundred degrees lower than hibonite. $\left({ }^{26} \mathrm{Al} /{ }^{27} \mathrm{Al}\right)_{0}$ values much lower than the "typical" value of $5 \times 10^{-5}$ have previously been observed in Allende inclusions B30 (LPW 1976a) and C1 (Esat et al. 1978). An important observation by Lorin and Christophe Michel-Levy (1978) using IPMA was that the $\left({ }^{26} \mathrm{Al} /{ }^{27} \mathrm{Al}\right)_{0}$ of some hibonites is $\sim 10^{-3}$, much higher than the typical value. These data show that ${ }^{26} \mathrm{Al}$ was widespread in silicate materials and that it may also have been present locally at exceedingly high levels in hibonite. The data for HAL represent the most extreme deviation from what is considered the typical value in a presumed very early condensate where large effects are anticipated. The lack of ${ }^{26} \mathrm{Al}$ in HAL hibonite and the presence of ${ }^{26} \mathrm{Al}$ in less refractory phases of other Allende inclusions require that (1) HAL crystallized much later than "typical" Allende inclusions; or (2) the distribution of ${ }^{26} \mathrm{Al}$ in the solar system was nonuniform, and HAL formed in a region where this nuclide was in low abundance. If the lack of ${ }^{26} \mathrm{Al}$ in $\mathrm{HAL}$ is due to time delay, then the crystallization of HAL took place after the formation of typical Allende inclusions by at least $5.6 \times 10^{6}$ years. This crystallization event must mark either the late onset of condensation from a gas at high temperature $\left(\gtrsim_{1700}\right.$ $\mathrm{K}$ ) or the cooling of the last residue of a droplet which was originally $\mathrm{Mg}$-rich and suffered large mass loss through evaporation during a late stage of intense heating. In either case, the simple model for sequential condensation in the solar nebula requires modification. Furthermore, the primitiveness of a sample cannot be inferred from mineralogical and chemical evidence alone. The alternative explanation for the lack of ${ }^{26} \mathrm{Al}$ in HAL is strong spatial variation of the $\mathrm{Al}$ isotopic composition similar to the isotopic heterogeneity observed for stable nuclides but greatly amplified. The $\mathrm{Ca}$ in HAL clearly has to come from a reservoir isotopically distinct from the parent reservoirs of typical Allende inclusions. If the heterogeneities are caused by the incomplete admixture of material from a special source which produced ${ }^{26} \mathrm{Al}$ and some stable nuclides including $\mathrm{Ca}$, then the variations in the ${ }^{26} \mathrm{Al} /{ }^{27} \mathrm{Al}$ ratio should be much larger than for the stable nuclides. This is because the stable nuclides from the last event would be diluted by those produced in all the previous events, while ${ }^{26} \mathrm{Al}$ would be entirely produced in the last event. A strong ${ }^{26} \mathrm{Al}$ heterogeneity could relax the constraint on the energetics for the ${ }^{26} \mathrm{Al}$ production in the solar system and permit a local proton irradiation model (Lee 1978). It would also limit the use of ${ }^{26} \mathrm{Al}$ as a heat source (Urey 1955) or ionization source (Consolmagno and Jokipii 1978) and as a chronometer.

No nuclear effect and only very small fractionation effects $(\leq 0.6 \%$ per amu) have been found among the terrestrial, lunar, and ordinary meteoritic samples studied by RPT (1978). Similar conclusions also apply to bulk Allende samples and to the usual $\mathrm{Ca}$-Al-rich inclusions, but extensive data are lacking (LPW 1978). LPW (1978) discovered in inclusions C1 and EK1-4-1 distinct $\mathrm{Ca}$ isotopic anomalies which are not dominated by fractionation processes (see Table 2 and Fig. 1). After decomposition, following the same procedure used for HAL, EK1-4-1 shows a large excess in ${ }^{48} \mathrm{Ca}$ and a small excess of ${ }^{42} \mathrm{Ca}$, while $\mathrm{C} 1$ shows a small deficiency in ${ }^{48} \mathrm{Ca}$. Also EK1-4-1 shows a fairly large fractionation effect favoring the light isotopes while C1 shows a very small fractionation effect favoring heavy isotopes. The interpretation has been that normal solar system $\mathrm{Ca}$ is the result of the mixing between uncontaminated bulk material which was deficient in ${ }^{48} \mathrm{Ca}$ and a special nuclear component consisting of ${ }^{48} \mathrm{Ca}$ and ${ }^{42} \mathrm{Ca}$ in the ratio of 2.3 . In this interpretation the deficiency of ${ }^{48} \mathrm{Ca}$ in $\mathrm{C} 1$ implies that the special nuclear component accounts for at least $3 \times 10^{-3}$ of ${ }^{48} \mathrm{Ca}$ presently in the solar system. It would be difficult to explain the data for HAL using the same nuclear component, since it is unlikely that the nuclear effect in ${ }^{48} \mathrm{Ca}$ is much larger than that for ${ }^{42} \mathrm{Ca}$ in this inclusion. Thus two independent nuclear 
components, one rich in ${ }^{42} \mathrm{Ca}$ and the other in ${ }^{48} \mathrm{Ca}$, may be required.

Based upon the isotopic and petrographic data, a plausible model for the evolution of HAL may be as follows: (1) condensation of refractory material which contains a substantial amount of normal $\mathrm{Mg}$ and some exotic nuclides from a special nucleosynthetic source including $\mathrm{Ca}$ and possibly also ${ }^{26} \mathrm{Al}$; (2) late heating $(\geq 1600 \mathrm{~K})$ of this material after the decay of ${ }^{26} \mathrm{Al}$ to evaporate away $\sim 50 \%$ of the $\mathrm{Ca}$ and most of the $\mathrm{Mg}$ to produce the fractionation; (3) crystallization of HAL containing hibonite and possibly also other refractory minerals; (4) reaction of HAL with surrounding gas to produce the black rim and the relatively volatile fine-grained exterior layers at low temperature $(<1000 \mathrm{~K})$; and $(5)$ fairly gentle incorporation into the Allende meteorite. In this model it is not clear whether the special nuclear component originated within the solar system or from a nearby star. It is necessary to assume that the gas for the back reaction is poor in $\mathrm{Ca}$, so that $\mathrm{Ca}$ anomalies are preserved in the volatile reaction products in the exterior layers, but that normal $\mathrm{Mg}$ was added. This model is somewhat similar to the one proposed for inclusion C1 (Esat $e t$ al. 1978).

The results from HAL emphasize that the relation between ${ }^{26} \mathrm{Al}$ and general nuclear anomalies is still an open question. They reinforce the scenario that the early solar system was chemically and isotopically heterogeneous and consisted of many distinct reservoirs. These reservoirs are the result of incomplete mixing between several nucleosynthetic components, and each has its own complicated thermal and chemical history. In this scenario there must also exist a mysterious linkage between certain efficient fractionation mechanisms and the preservation (or generation) of isotopically anomalous material. The discovery of HAL demonstrates that isotopically anomalous material is sufficiently abundant and can be located by using peculiar mineralogy and chemistry, in addition to large isotopic fractionation, as signposts.

We thank E. Anders and R. Lewis for help in the excavation of HAL. D. A. Papanastassiou's gracious aid with the mass spectrometry and his sagacious suggestions were essential to the completion of this work. T. L. gratefully acknowledges the warm hospitality from the inmates of the Lunatic Asylum while he was a Guest Investigator there and acknowledges the support from R. N. Clayton and T. K. Mayeda of the Fermi Institute. This work was supported in part by NSF grant 76-83685 and NASA grant NGL05-002-188 at the California Institute of Technology and also by funds from the Robert R. McCormick Trust and NSF grant EAR 74-19038 at the University of Chicago.

\section{REFERENCES}

Allen, J. M., Grossman, L., Lee, T., and Wasserburg, G. J. 1979, in preparation.

Blander, M., and Fuchs, L. H. 1975, Geochim. Cosmochim. Acta, 38, 1605 .

Bradley, J. G., Huneke, J. C., and Wasserburg, G. J. 1977, J. Geophys. Res., 83, 244.

Consolmagno, G. J., and Jokipii, J. R. 1978, Proc. Tucson Conf. Protostars Planets, ed. T. Gehrels, in press.

Davis, A. M., Tanaka, T., Grossman, L., Lee, T., and Wasserburg, G. J. 1979, in preparation.

Esat, T. M., Lee, T., Papanastassiou, D. A., and Wasserburg, G. J. 1978, Geophys. Res. Letters, 5, 807.

Hutcheon, I. D., Steele, I. M., Clayton, R. N., and Smith, J. V. 1978, Meteoritics, 13.

Keil, K., and Fuchs, L. H. 1971. Earth Planet. Sci. Letters, 12, 184.

Lee, T. 1978, $A p$. J., 224, 217.

Lee, T., and Papanastassiou, D. A. 1974, Geophys. Res. Letters, 1, 225 (LP)

Lee, T., Papanastassiou, D. A., and Wasserburg, G. J. 1976a, Geophys. Res. Letters, 3, 109 (LPW 1976a).

1976b, Trans. Am. Geophys. Union, 57, 278 (LPW 1976b). 1977a, Ap.J. (Letters), 211, L107 (LPW 1977a).

1977b, Geochim. Cosmochim. Acta, 41, 1473 (LPW 1977b).
- 1978, Ap. J. (Letters), 220, L21 (LPW 1978).

Lorin, J. C., and Christophe Michel-Levy, M. 1978, Proc. 4th Internat. Conf. Geochro. Cosmochro. Isotope Geo. (Denver: US Geological Survey), p. 257.

McCulloch, M. T., and Wasserburg, G. J. 1978, Ap. J. (Letters), 220, L15.

Papanastassiou, D. A., Lee, T., and Wasserburg, G. J. 1977, in Comets, Asteroids, Meteorites: Interrelations, Evolution and Origins, IAU Colloquium No. 39, ed. A. H. Delsemme (Toledo: University of Toledo Press), p. 343 (PLW).

Rayleigh, J. W. S. 1896, Phil. Mag., 42, 493.

Russell, W. A., Papanastassiou, D. A., and Tombrello, T. A. 1978, Geochim. Cosmochim. Acta, 42, 1075 (RPT).

Schramm, D. N., Tera, F., and Wasserburg, G. J. 1970, Earth Planet. Sci. Letters, 10, 44

Urey, H. C. 1955, Proc. Nat. Acad. Sci., 41, 127.

Wark, D. A., and Lovering, J. F. 1977, Lunar Science VII (Houston: Lunar Science Institute), p. 976.

Wasserburg, G. J., Lee, T., and Papanastassiou, D. A. 1976, Meteoritics, 11, 384 (WLP).

Wasserburg, G. J., Papanastassiou, D. A., Nenow, E. V., and Bauman, C. A. 1969, Rev. Sci. Instr., 40, 288.

Typhoon LeE: Enrico Fermi Institute, University of Chicago, 933 E. 56th St., Chicago, IL 60637

William A. Russell and Gerald J. Wasserburg: Division of Geological and Planetary Sciences, 170-25, California Institute of Technology, Pasadena, CA 91125 